\title{
IPCAI 2018 Special Issue: Information Processing for Computer-Assisted Interventions, 9th International Conference 2018-Part 1
}

\author{
Pascal Fallavollita ${ }^{2} \cdot$ Etsuko Kobayashi $^{3} \cdot$ Stefanie Speidel $^{1}$
}

Published online: 7 May 2018

(c) CARS 2018

It is a pleasure to present this special issue of IJCARS that includes the proceedings of the 9th edition of the International Conference on Information Processing for ComputerAssisted Interventions (IPCAI). IPCAI will be held at Hotel $\mathrm{NH}$ Collection Berlin Mitte in Berlin, Germany, on June 20-21, 2018, in conjunction with the Computer Assisted Radiology and Surgery (CARS) Congress.

The IPCAI series of meetings was created in 2010 in Geneva, as a forum to discuss the latest developments in Computer-Assisted Interventions (CAI). CAI is a field of research and practice, where medical interventions are supported by computer-based tools and methodologies. CAI systems enable precise, safe and minimally invasive medical interventions by providing enhanced planning, real-time imaging, instrument guidance and visualization, as well as situation awareness and cognition. To promote translational research, the IPCAI meetings seek to showcase papers presenting novel technical algorithms and theory, clinical applications and hardware as well as software systems and their validation.

With substantial support from the scientific community, IPCAI has consistently grown in the past 8 years in terms of

Stefanie Speidel

stefanie.speidel@nct-dresden.de

Pascal Fallavollita

pfallavo@uottawa.ca

Etsuko Kobayashi

kobayashi.etsuko@twmu.ac.jp

1 Division Translational Surgical Oncology, National Center for Tumor Diseases (NCT), Partner Site Dresden, Fetscherstraße 74, PF 41, 01307 Dresden, Germany

2 Interdisciplinary School of Health Sciences, Faculty of Health Sciences, University of Ottawa, 200 Lees Avenue, Ottawa, ON K1S 5S9, Canada

3 Faculty of Advanced Techno-Surgery (FATS), Institute of Advanced Biomedical Engineering and Science, Tokyo Women's Medical University, 8-1 Kawada-cho, Shinjuku-ku, Tokyo 162-8666, Japan the number of submissions and the presented manuscripts. This year IPCAI has received 85 manuscripts spanning a strong geographic representation from Europe, North America and Asia.

Unlike most conferences, manuscripts submitted to IPCAI 2018 are expected to have journal quality, as our goal is to publish all accepted manuscripts in this special volume of the International Journal of Computer Assisted Radiology and Surgery (IJCARS). The review process was rigorous and involved evaluation of each manuscript by two Area Chairs and at least three external reviewers. Authors were able to provide a revised version of their manuscript and a response to reviewers based on the reviewers' comments. The revised manuscripts were subject to an additional review process ensuring that all reviewers' critiques were properly addressed and that the quality was appropriate for journal publication. Prior to acceptance, manuscripts were discussed by the Program Chairs, Program Board members and the Conference chairs.

In total, 40 manuscripts were accepted for presentation at IPCAI 2018, with 14 of them appearing in this issue. The remaining manuscripts will be published in subsequent IJCARS Special Issues, dependent upon the authors completing the appropriate revisions. The accepted manuscripts reflect the breadth of key topics in CAI and include: (i) Endoscopy and Surgical Training; (ii) Interventional Ultrasound; (iii) Preoperative Planning and Simulation; (iv) Intraoperative Navigation and Registration; (v) Image Processing and Instrumentation for CAI; and (vi) Medical Robotics.

The 2018 format of IPCAI is designed to encourage interaction and allow time for constructive discussion. All authors of accepted manuscripts were asked to deliver a short, 5min platform presentation. In addition, each manuscript was presented as a poster during an interactive session. The conference delegates will be asked to vote for papers that they would like to see discussed further. The papers with the highest number of votes will be presented in extended format 
followed by questions from the attendees and the committee members.

We would like to take this opportunity to thank the Area Chairs, the Program Board and all of the reviewers for their dedication to maintaining the high quality of IPCAI and for selecting outstanding manuscripts that will no doubt stimulate exciting discussions at the meeting.

We acknowledge our generous sponsors for their continued support over the years that has enabled us to recognize both IPCAI authors and reviewers for their much deserved dedication and scientific enthusiasm through several paper awards. The awards include the Outstanding Reviewer Award, Young Investigator Travel Award, Bench-to-Bedside Award and Best Paper Award, all made possible through their generous sponsorship. A big thank you on behalf of all awardees!

Last but not least, we also thank the CARS Congress Organizers and Editorial Office for their help and support with the logistics and with the IJCARS manuscript management and review process.

We are grateful to all authors for choosing to submit their manuscripts to IPCAI and hope that the readers will enjoy this special issue. The continued success of IPCAI demands our ongoing commitment.

Pascal Fallavollita, Etsuko Kobayashi, Stefanie Speidel (Program Chairs)

May 2018

\section{Organization}

\section{Program Chairs}

Pascal Fallavollita, University of Ottawa, Canada

Etsuko Kobayashi, Tokyo Women's Medical University, Japan

Stefanie Speidel, National Center for Tumor Diseases, Dresden, Germany

\section{General Chairs}

Kensaku Mori, Nagoya University, Japan

Parvin Mousavi, Queen's University, Canada

Danail Stoyanov, University College London, UK

\section{Area Chairs-Academia}

Elvis Chen, Western University, Canada

Sandrine DeRibaupierre, Western Ontario, Canada

Adrien Desjardins, University College London, UK

Stefanie Demirci, Technical University of Munich, Germany

Caroline Essert, University of Strasbourg, France

Gabor Fichtinger, Queen's University, Canada
Stamatia Giannarou, Imperial College London, UK

Orcun Goksel, ETH, Switzerland

Christoph Hennersperger, Technical University of Munich, Germany

Chui Chee Kong, National University of Singapore, Singapore

Sun-Lin Lee, Imperial College London, UK

Cristian A. Linte, Rochester Institute of Technology, USA

Ken Masamune, Tokyo Women's Medical University, Japan

Nicolas Padoy, University of Strasbourg, France

Amber L. Simpson, Memorial Sloan Kettering Cancer Center, USA

Raphael Sznitman, University of Bern, Switzerland

Siyang Zuo, Tianjin University, China

Area Chairs-Industry

Mahdi Azizian, Intuitive Surgical, USA

Peter Mountney, Siemens Healthineers, UK

Amir Tahmasebi, Philips Research North America

Andrew Wiles, Northern Digital Inc., Canada

\section{Program Board}

David Hawkes, University College London, UK

Nassir Navab, Technical University of Munich, Germany \& Johns Hopkins University, USA

Tim Salcudean, University of British Columbia, Canada

Russell Taylor, Johns Hopkins University, USA

\section{Steering Committee}

Purang Abolmaesumi, University of British Columbia, Canada

Kevin Cleary, Children's National Medical Center, USA

Gabor Fichtinger, Queen's University, Canada

Makoto Hashizume, Kyushu University, Japan

David Hawkes, University College London, UK

Pierre Jannin, Université de Rennes, France

Leo Joskowicz, The Hebrew University of Jerusalem, Israel

Ron Kikinis, Brigham and Women's Hospital, USA

Heinz Lemke, Technical University of Berlin \& IFCARS

Kensaku Mori, Nagoya University, Japan

Nassir Navab, Technical University of Munich, Germany \&

Johns Hopkins University, USA

Terry Peters, Western University, Canada

Ichiro Sakuma, The University of Tokyo, Japan

Tim Salcudean, University of British Columbia, Canada

Gábor Székely, ETH Zentrum, Switzerland

Russell Taylor, Johns Hopkins University, USA

Guang-Zhong Yang, Imperial College, London, UK 


\section{Reviewers}

Abinahed Julien

Abolmaesumi Purang

Ahmadi Ahmad

Albarqouni Shadi

Alles Erwin

Anglin Carolyn

Arata Jumpei

Ayyildiz Mehmet

Azizi Shekoofeh

Balicki Marcin

Bartoli Adrien

Baxter John

Bello Fernando

Berger Marie-Odile

Bertero Dario

Bharat Shyam

Billings Seth

Black David

Bodenstedt Sebastian

Bouget David

Bui Mai

Burgert Oliver

Cahill Nathan

Camara Mafalda

Capobianco Antonio

Che Yuhang

Chen Thomas

Chi Wenqiang

Chiang Patricia

Chinzei Kiyoyuki

Chng Chin-Boon

Clarkson Matt

Collins Jarrod A.

Collins Toby

Constantinescu Mihaela

Cotin Stephane

Dawant Benoit

De Momi Elena

De Zanet Sandro

Dehghan Ehsan

Delingette Herve

Deshmukh Deshmukh Nishikant

Deshmukh Nishikant

Desvignes Michel

Dogramadzi Sanja

Drouin Simon

Duchateau Nicolas

Duong Luc

Eck Ulrich

Elson Daniel

Fenster Aaron

Figl Michael
Finlay Malcolm

Fitzpatrick Mike

Forestier Germain

Fountoukidou Tatiana

Freiman Moti

Freysinger Wolfgang

Friebe Michael

Frisch Benjamin

Fu Yabo

Fuerst Bernhard

Funke Isabel

Garcia Guevara Jaime

Goceri Evgin

Golkar Ehsan

Gong Yuanhao

Groehl Janek

Gu Yun

Guo Yi

Göbl Rüdiger

Habert Severine

Hacihaliloglu Ilker

Haidegger Tamas

Hariharan Sai Gokul

Hashizume Makoto

Hefny Mohamed

Hodgson Antony

Howe Robert

Hummel Johann

Imani Farhad

Jain Ameet

Jannin Pierre

Jayarathne Uditha

Jiang Shan

Joskowicz Leo

Kapoor Ankur

Katic Darko

Kazanzides Peter

Kazmitcheff Guillaume

Kersten-Oertel Marta

Khalaji Iman

King Andrew

Kiryati Nahum

Kojchev Risto

Kotwal Alankar

Kurmann Thomas

Kwok Ka-Wai

lamata pablo

Lango Thomas

Lasso Andras

Lentes Beatrice

Liu Liu Jindong

Loy Rodas Nicolas

Luo Xiongbiao 
López-Linares Román Karen

Ma Burton

Ma Jiayao

Malpani Anand

Marmol Andres

Marquering Henk

McLeod Jonathan

Miao Shun

Mohareri Omid

Moradi Mehdi

Mori Kensaku

Mousavi Parvin

Nair Dhanya

Nakamura Ryoichi

Naoki Tomii

Neumuth Neumuth Thomas

Niederer Steven

Nir Guy

Ohtaki Yasuaki

Otake Yoshito

Ozdemir Firat

Pace Danielle

Paul Thienphrapa

Peters Jochen

Peters Terry

Pfeiffer Micha

Pheiffer Tom

Plantefeve Rosalie

Pratondo Agus

Prince Jerry

Quellec Gwenole

Raisin Philippe

Rasoulian Abtin

Regensburger Alois

Reichard Daniel

Rettmann Maryam

Rivaz Hassan

Rohling Robert

Sakuma Ichiro

Salcudean Tim

Salehi Mehrdad

Samei Golnoosh

Schadewaldt Nicole

Schmidt-Richberg Alexander

Schweikard Achim

Seitel Alexander
Sethuraman Shriram

Shamir Ruby

Singla Rohit

Sinha Ayushi

Sitek Arkadiusz

Stoyanov Danail

Strobel Norbert

Tan Xiaoyu

Tangen Geir

Tanner Christine

Tavares João Manuel RS

Taylor Russ

Thienphrapa Paul

Thompson Stephen

Toth Daniel

Tsui Po-Hsiang

Twinanda Andru Putra

Uemura Munenori

Ungi Tamas

van Walsum Theo

Vercauteren Tom

Virga Salvatore

Visentini-Scarzanella Marco

Voros Sandrine

Wang Junchen

Waterbury Andrew

Weber Stefan

Weese Juergen

Xia Xia Wenyao

Xia Wenfeng

Xing Yuan

Yan Pingkun

Yang Tao

Yaniv Ziv

Ye Menglong

Zettinig Oliver

Zhang Lei

Zhang Lin

Zhang Mei

Zheng Guoyan

Zuluaga Maria

\section{Compliance with ethical standards}

Conflict of Interest The authors declare that they have no conflict of interest. 